\title{
Predicting mortality in patients with non-ischemic dilative cardiomyopathy: Potential of extracellular volume imaging by cardiovascular magnetic resonance
}

\author{
Katharina Koopmann ${ }^{\text {1*, Ulf K Radunski', Sebastian Bohnen', Lukas Radziwolek', Gunnar Lund }{ }^{2} \text {, Christian Stehning }}{ }^{3}$, \\ Gerhard Adam², Stefan Blankenberg', Kai Muellerleile ${ }^{1}$
}

From 17th Annual SCMR Scientific Sessions

New Orleans, LA, USA. 16-19 January 2014

\section{Background}

Predicting mortality in patients with non-ischemic dilated cardiomyopathy (NIDCM) is currently demanding and requires complex models. Assessing the extracellular volume fraction (ECV) by T1-mapping cardiovascular magnetic resonance (CMR) is an attractive approach to quantify myocardial injury as a potential predictor of adverse events in these patients. This study evaluated ECV imaging by T1-mapping CMR in comparison with the established "Seattle Heart Failure Model" (SHFM; http://www.seattleheartfailuremodel.org).

\section{Methods}

This study included 50 patients with heart failure and reduced left ventricular (LV) ejection fraction due to non-ischemic cardiomyopathy. The SHFM was used to estimate 1, 2 and 5 year mortalities as well as life expectancy, respectively. T1 quantification was performed at 1.5 Tesla using the modified Look-Locker inversionrecovery (MOLLI) sequence on 3 short-axes before and 15 minutes after administration of $0.075 \mathrm{mmol} / \mathrm{kg}$ gadolinium-BOPTA. Global myocardial ECV was then calculated from native and post-contrast $\mathrm{T} 1$ maps generated by a dedicated plug-in written for the OsiriX software, respectively.

\section{Results}

Median predicted 1-, 2- and 5-year mortalities were $4 \%$ (interquartile range $2-6 \%$ ), $7 \%$ (interquartile range $5-12 \%$ ) and $16 \%$ (interquartile range $12-28 \%$ ) in the study population, respectively. Median estimated life expectancy was 13 years (interquartile range 9-15 years). A significant correlation was found between global myocardial ECV and predicted $1-(\mathrm{r}=0.47 ; \mathrm{p}<0.001), 2-(\mathrm{r}=0.46$; $\mathrm{p}<0.001)$ and 5-year mortality $(\mathrm{r}=0.46 ; \mathrm{p}<0.001)$, respectively. Furthermore, a significant inverse correlation was found between global myocardial ECV and predicted life expectancy $(\mathrm{r}=-0.37 ; \mathrm{p}<0.01)$.

\section{Conclusions}

Myocardial injury as quantified by ECV-imaging correlates well with predicted mortality and has great potential to improve risk stratification in patients with heart failure due to NIDCM.

\section{Funding}

No funding.

\section{Authors' details}

${ }^{1}$ Cardiology, University Heart Center, Hamburg, Germany. ${ }^{2}$ Radiology, University Medical Center, Hamburg, Germany. ${ }^{3}$ Philips Research, Hamburg, Germany.

Published: 16 January 2014

${ }^{1}$ Cardiology, University Heart Center, Hamburg, Germany

Full list of author information is available at the end of the article

doi:10.1186/1532-429X-16-S1-P88

Cite this article as: Koopmann et al.: Predicting mortality in patients with non-ischemic dilative cardiomyopathy: Potential of extracellular volume imaging by cardiovascular magnetic resonance. Journal of Cardiovascular Magnetic Resonance 2014 16(Suppl 1):P88. 